\title{
Post Covid-19 Infection Presenting as Rhino-Orbital Mycosis
}

\author{
Richa Garg $^{1} \cdot$ Sandeep Bharangar $^{2} \cdot$ Sunil Gupta $^{3} \cdot$ Samiksha Bhardwaj $^{4}$
}

Received: 15 June 2021 / Accepted: 27 June 2021 / Published online: 14 July 2021

(C) Association of Otolaryngologists of India 2021

\begin{abstract}
Rhino-orbital mycosis has been recently recognised as one of the sequelae in COVID-19 recovered patients. In India, detection of mucormycosis is declared as notifiable disease. In this article, the authors aim to describe the characteristics of patients presenting with post covid fungal infection which could be detected on $10 \%$ potassium hydroxide $(\mathrm{KOH})$ wet mount and Giemsa stain put on crush biopsy smear. We describe 10 COVID-19 recovered patients admitted to ENT department of the hospital during second wave of COVID-19 infection. They presented with post covid fungal sinusitis and ophthalmic complications and planned for surgery. $\mathrm{KOH}$ mount and Giemsa stain were used for possible opinion and confirmed by culture. The observations were described in mean and percentages. All ten (100\%) COVID-19 recovered patients were previously diagnosed with type 2 diabetes mellitus (DM) for 2-11 years. All 10 patients (100\%) were given oral or intravenous corticosteroids for mean of 21 days (3 weeks - till presentation to ENT department). Simple procedures with $10 \% \mathrm{KOH}$ mount and Giemsa stain could detect fungal hypae in all the cases and could provide possible opinion in 9 of $10(90 \%)$ cases for timely management of the patients. The authors hypothesize that
\end{abstract}

Richa Garg

richag24@gmail.com

1 Consultant Pathologist, Crest Hospital, Moradabad, Uttar Pradesh, India

2 Consultant Otolaryngologist, Crest Hospital, Moradabad, Uttar Pradesh, India

3 Consultant Opthalmologist, Sahu Rameshwar Saran Hospital and Research Centre, Moradabad, Uttar Pradesh, India

4 Consultant Radiologist, Crest Hospital, Moradabad, Uttar Pradesh, India uncontrolled DM and prolonged use of corticosteroids may act as culprits of rhino-orbital mycosis in COVID-19 recovered patients. Simple and routine $10 \% \mathrm{KOH}$ mount and Giemsa stain may provide early opinion of fungal hypae to ensure quick management and survival of the patients.

Keywords Covid-19 - Diabetes-mellitus · Corticosteroids . Mucormycosis $\cdot \mathrm{KOH} \cdot$ Giemsa

\section{Introduction}

Coronavirus disease is caused by severe acute respiratory syndrome coronavirus 2 (SARS-COV2). The infection spreaded across the continents resulting in pandemic [1]. The first wave hit the patients more severely with comorbidities like diabetes mellitus (DM), heart problems, body mass index $>40 \mathrm{~kg} / \mathrm{m}^{2}$ and age $>60$ years [2]. The second wave of COVID-19 is more severe and brought healthcare system to use plasma therapy, remdesiver injections, corticosteroids and oxygen cylinders [1]. The clinical manifestations of patients with COVID-19 infection are common in both waves: fever, cough, fatique and shortness of breath [1]. The second wave has also came across features like headache, localised pain, nasal discharge, sinusitis, orbital cellulitis and dimuniation of vision due to rhino-orbital fungal infection occuring few weeks after recovery of COVID-19 patients [3-5]. Fungal infection mainly affects people on medication due to their reduced ability to fight environmental pathogens [3-6]. The detection of mucormycosis- an uncommon opportunistic invasive fungal infection is declared as notifiable disease under Epidemic Diseases Act 1897 on 19 May 2021 in India. Mucormycosis is caused by species of Rhizopus, 
Mucor and Absidia [7]. It may be a terminal complication of uncontrolled diabetes. The incidence of mucormycosis has increased considerably as a result of widespread use of steroids, antibiotics and antimetabolites [4-7]. The infection starts in the upper respiratory tract or nose by inhalation of spores and may invade adjacent tissues like orbit, sinuses and brain [7]. Till date approximately 28,200 cases of mucormycosis have been recorded in India [8]. Warning signs of rhino-orbital fungal infection include sinusitis, blurred vision, redness of eye, blocked nose, local pain, blackish discoloration over bridge of nose and palate on clinical examination [3-5]. The other opportunstic fungal infections reported in COVID-19 patients are oropharyngeal candidiasis and respiratory tract aspergillosis [9]. The commonly used fungal stain is $\mathrm{KOH}$ as wet mount to demonstrate fungal hypae on the nasopharyngeal swab [7]. The present article demonstrates the characteristics of COVID-19 recovered patients presenting with clinical features suspicious of rhino-orbital fungal infection and the use of routine $\mathrm{KOH}$ mount and Giemsa stain on crush biopsy smear for early detection and management.

\section{Methods}

The clinical details of 10 patients who were recovered from COVID-19 infection and admitted to ENT department in the hospital in month of May 2021 were noted down (Table, (Fig. 1a). Imaging was done in each case as computed tomography (CT) or magnetic resonance imaging (Fig. 1b). Nasal endoscopy was done in all 10 cases (Fig. 1c). The cases with intracranial extension on imaging were referred to higher centre and not considered. Advanced endoscopic sinus surgery (AESS) was planned in all 10 cases after taking written consent. The operated specimen was labelled as nasal/paranasal mucosa and sent to lab in normal saline for fungal microscopy (Fig. 2a). Two crush smears were made from the blackish area of the specimen (Fig. 2b) and stained with $10 \% \mathrm{KOH}$ and giemsa stains respectively (Fig. 2c-d). Microscopy was done first at 20X and then 40X. Broad, aseptate to sparsely septate, wide angled branching fungal hypae were considered belonging to order mucorales and thin septate acute angled branching fungal hypae were suggestive of aspergillus. [7], The rest of the biopsy specimen was outsourced for fungal culture. The observations and results are expressed in mean and percentages.

\section{Observations}

The clinical features of 10 patients are tabulated in table (Table 1). In the present article, 6 of $10(60 \%)$ were male and 4 of $10(40 \%)$ were females patients. The mean age of the patients admitted was 53.3 (28-70years). The predominant symptom was headache seen in 10 of 10 $(100 \%)$ patients, diminution of vision was observed in 3 of $10(30 \%)$ patients. Other complaints were cheek swelling, nasal obstruction, nasal discharge and localised pain (Fig. 1a). All 10 cases were not vaccinated. All patients were COVID-19 positive reported either by reverse transcriptase polymerase chain reaction (rtpcr) positive seen in 6 of 10 cases $(60 \%)$ or antigen positive observed in 4 of 10 $(40 \%)$ of patients. The mean number of days with patients presenting with rhino-orbital fungal infection was 24.6 days (range 22-28 days) from their COVID -19 positive status. Imaging as CT or MRI was done in all the cases which showed infective sinusitis in 7 of 10 cases $(70 \%)$. The predominant risk factor was DM type -II seen in 10 of 10 cases $(100 \%)$ present for a mean of 4.5 years (range 2-11 years). The mean random blood glucose level at presentation to ENT department was $299.4 \mathrm{mg} / \mathrm{dl}$ (range: $185-447 \mathrm{mg} / \mathrm{dl})$. All patients $(100 \%)$ were taking oral hypoglycemic drugs and 4 of $10(40 \%)$ were using insulin also. All 10 patients (100\%) were given oral corticosteroids as part of management of COVID-19 infection for mean of 24 days (range: 21 days- till presentation to ENT department). The administered doses were methylprednisolone (MP) 16mg TDS or dexamethasone (DX) 8mg BD with tapering doses over weeks. The oxygen saturation of the patients during COVID-19 positivity ranged from 90 to 99\%. However, none of the patients required ventilator support or admission in ICU. 3 of 10 (30\%) patients required 2litre-8litre external oxygen support through oxygen cylinders for 5-7 days. All 10 of 10 (100\%) patients underwent nasal endoscopy and later were planned for AESS as treatment modality. In all the patients, the operative mucosal specimen was labelled and sent to pathology lab for fungal microscopy and culture. On microscopy with $\mathrm{KOH}$ mount and Giemsa stain, broad aseptate to sparsely septate fungal hypae possibly belonging to order mucorales/mucormycosis were noted in 7 of $10(70 \%)$ cases (Fig. 3a-b) and thin acute angled, branched septate fungal hypae possibly belonging to family aspergillus observed in 2 of 10 (20\%) cases (Fig. 3c-d). Predominantly thin septate and occasional broad sparsely septate fungal hypae seen in 1 of $10(10 \%)$ cases and was reported as possibly mixed infection (Fig. 3e). The reports could later be confirmed by fungal culture as 7 cases of mucormycosis $(70 \%)$ and 3 aspergillus (30\%). So, in 9 of 10 cases $(90 \%), \mathrm{KOH}$ mount and giemsa stain could provide opinion correlating with fungal culture. All the patients were given antibiotics and antihistaminincs post surgery and advised for nasal douching. 3 of 7 patients $42.8 \%$ diagnosed with mucormycosis were given liposomal amphotericin B $(5 \mathrm{mg} /$ $\mathrm{kg} /$ day) and 1 of 3 patients (33.3\%) diagnosed with aspergillosis was given oral posaconazole in a dose of 50 


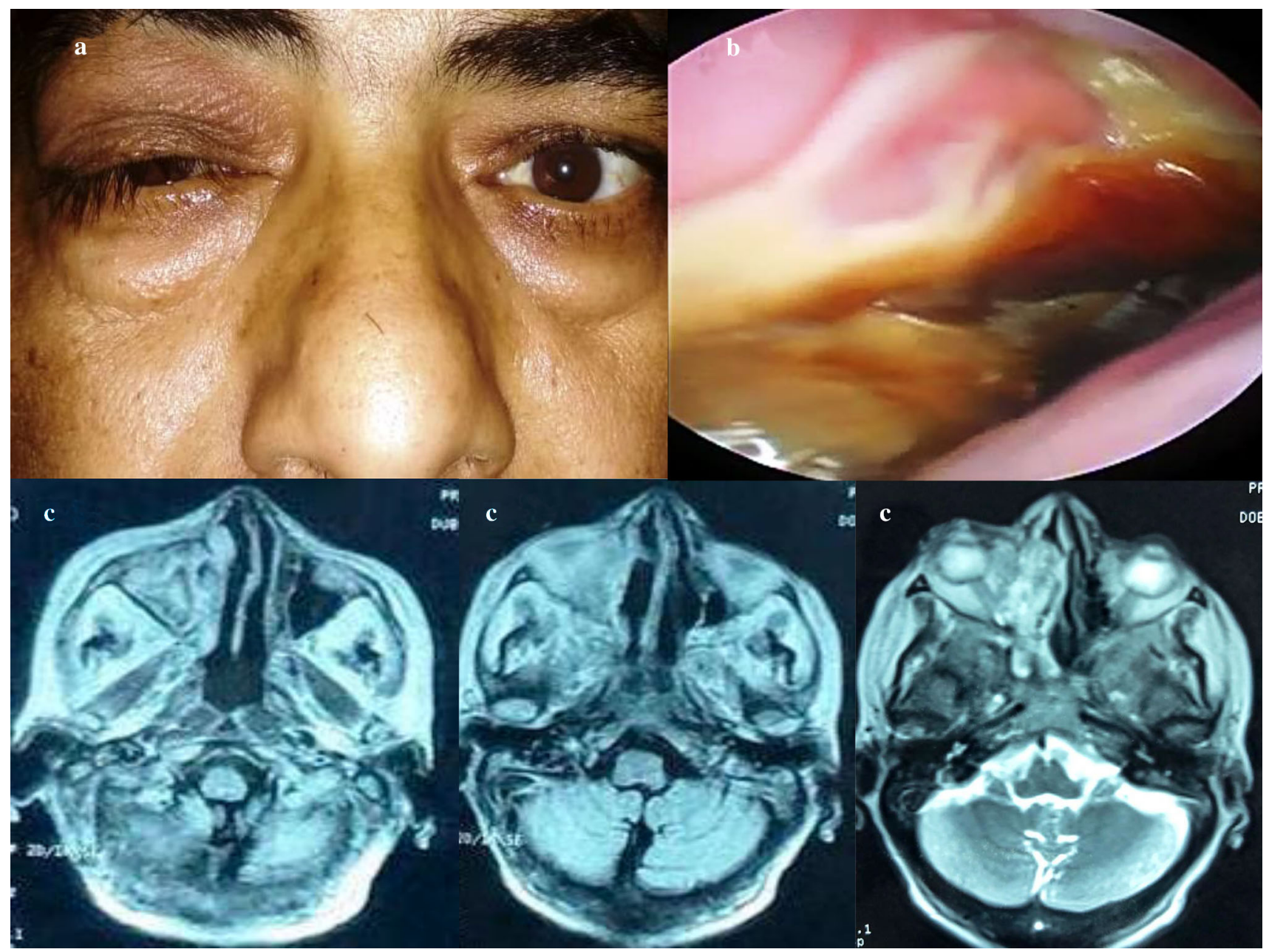

Fig. 1 a Patient presenting with nasal blockage, local pain and orbital swelling. b Nasal endsoscopy showing pale mucosa with black necrotic eschar. c CT scan showing rhino-orbital mucosal thickening, edema and mass collection suggestive of infective etiology

mg TDS for 5 days at the time of resolution. The opthalmoplegia regained back after AESS in all 3 of $3(100 \%)$ of patients presenting with dimuniation of vision with better perception of light after 5-7 days of surgery. None of the deaths reported so far.

\section{Discussion}

Coronavirus disease is caused by SARS-CoV2 virus which has infected approximately 174 million people globally so far. According to WHO, India has reported 29,359,155 confirmed cases of COVID-19 infection with 367,081 deaths from 3 January 2020 to 12 June 2021 [10]. COVID-19 infection has hit India badly during the second wave. On May 7th 2021, India has recorded its ever highest 4.14 lakhs COVID-19 cases in a single day [10]. The most common symptoms of covid-19 remain fever, dry cough and fatique. People above 60yeras of age, with heart and lung problems, obese, diabetic are at higher risk of becoming critically ill. People who have had COVID-19 infection whether hospitalized or not continue to experience fatique, respiratory and neurological symptoms for some time [10].

The COVID- 19 infection dysregulates CD4 + and CD8 + cells disturbing the innate immunity which increases the chances of secondary fungal and bacterial infections [11, 12]. In our article, all the 10 patients were COVID- 19 positive (22-28 days) before detection of fungal infection. COVID-19 patients have more pro-inflammatory (IL-1, IL-2, IL-6, tumor necrosis factor- alpha) cytokine levels and less $\mathrm{CD}_{4}$ interferon-gamma expression which increases the risk of invasive fungal infection in them.

Mucormycosis and aspergillosis are important opportunstic systemic mycoses. Their spores are inhaled in immunocompromised states which germinate in nasal cavity, paranasal sinuses, lung orbit and brain which may lead to death [7, 12]. All our 10 patients were $2-11$ years old diabetic. Diabetes is a state of immunosuppression. 


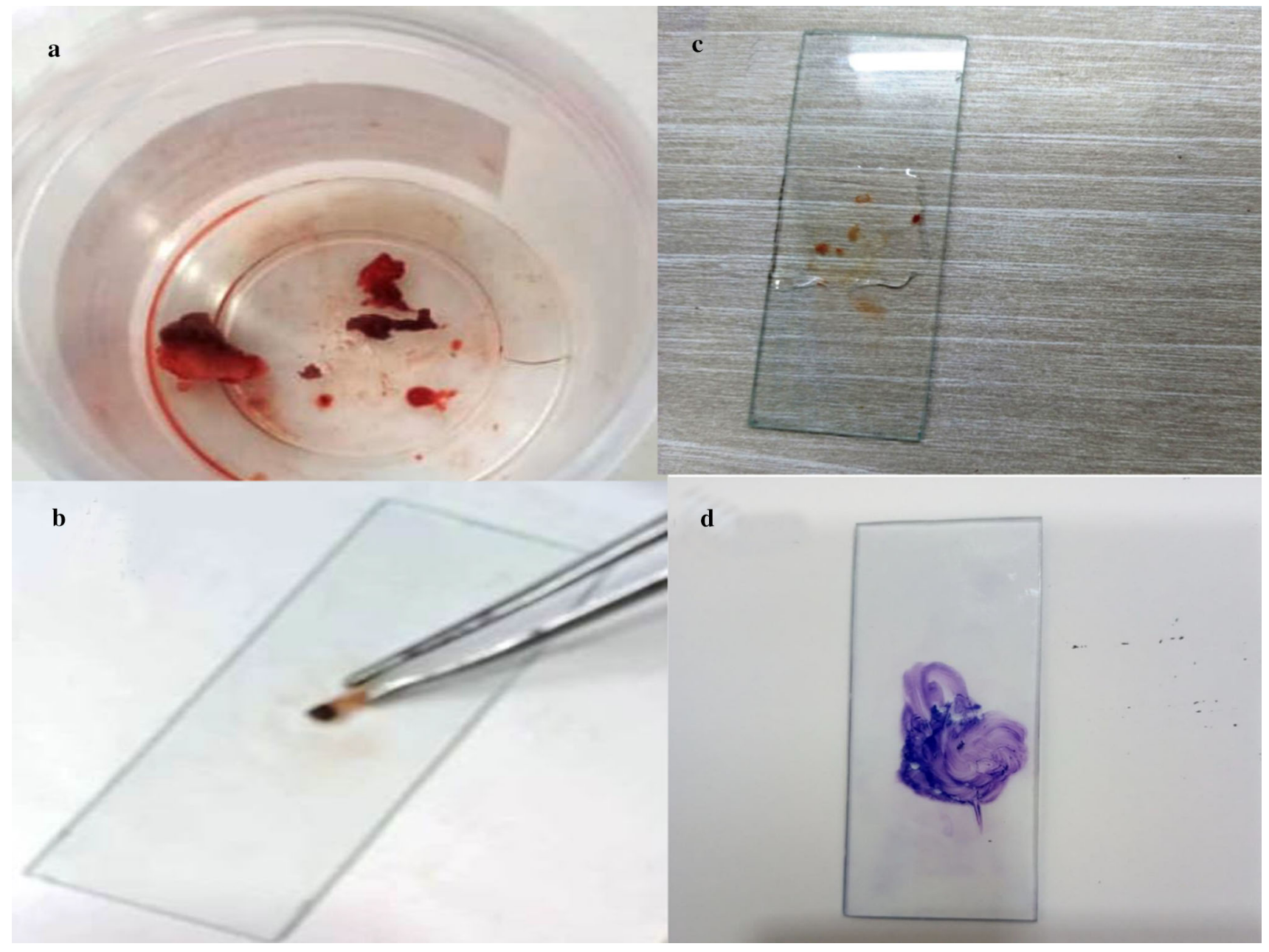

Fig. 2 a Blackish to pinkish mucosal specimen. b smear preparation from blackish area of specimen. c Smear with $10 \%$ KOH wet mount. d Smear stained with Giemsa stain

Hyperglycemia in diabetic patients distrupts immune response and affects cellular immunity, causes defective phagocytosis, suppresses cytokine production and results in failure to attack microbes causing invasion by secondary fungal infections [13]. COVID-19 infection virus (SARSCov 2) itself causes hperglycemia as the virus damages the pancreatic islets due to cytokine storm [10, 14]. So, the blood glucose levels increases significantly in already diabetic patients when infected with COVID-19. It is recommended by WHO to monitor blood glucose levels twice daily for 3-4 days in COVID-19 patients and to start insulin if pre-meal blood glucose level is $\geq 180 \mathrm{mg} / \mathrm{dl}$ or post-meal blood glucose $\geq 250 \mathrm{mg} / \mathrm{dl}$ to avoid uncontrolled hyperglycemia [10]. In our article, 7 of 10 cases were confirmed as mucormycosis and 3 of 10 cases as aspergillus. The authors noted that mucormycosis which was seen in $70 \%$ of cases had random blood sugar level more than $300 \mathrm{mg} / \mathrm{dl}$ at presentation while in $30 \%$ cases noted as aspergillus had blood sugar level less than $300 \mathrm{mg} / \mathrm{dl}$. So, we may hypothesize that mucorales need more sugar level for their growth. However, more research needs to be done for its support.

The second wave of COVID-19 infection caused extensive use of corticostroids as a part of COVID-19 management either as hospital care or at home under online supervision. Glucocorticosteroids have anti-inflammatory and immunosuppressive effects.

Steroids antagonizes IL-1, IL-6, TNF and microbicidal activities of activated macrophages [15].

Some studies have shown that prolonged use of corticosteroids to control cytokine storm contributed to more susceptibility to fungal infection due to their immunosuppressive behaviour [11, 12, 14, 15]. Steroids also affects glycemic status causing hyperglycemia. According to WHO, if COVID patient is initiated on steroids, blood glucose monitoring should be done as before breakfast, lunch and dinner and after dinner also [10]. In our article, all 10 of 10 patients have used high doses glucocorticosteroids (Table 1) at least for 3 weeks and 4 of 10 were taking steroids till they were presented to ENT department. 


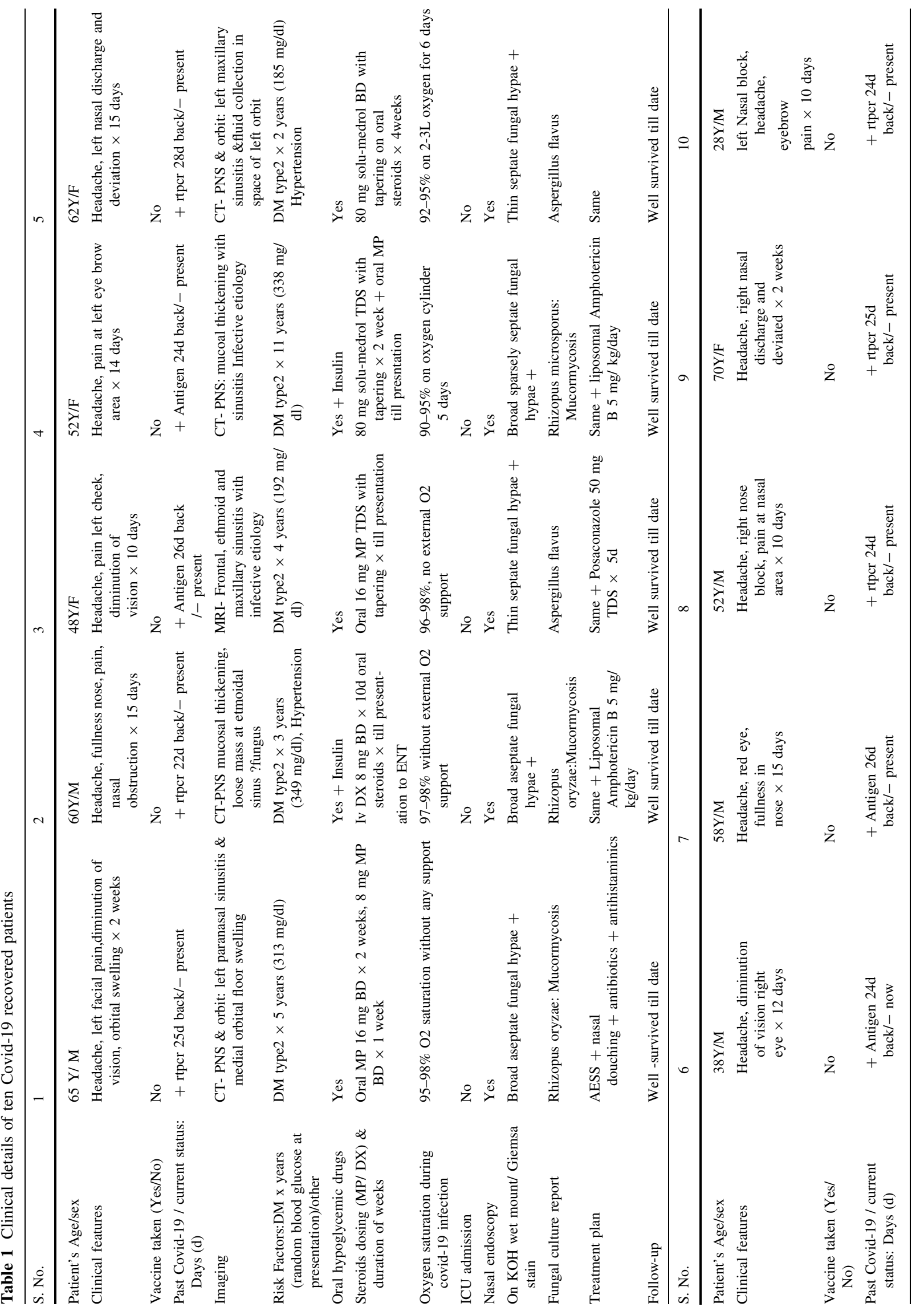




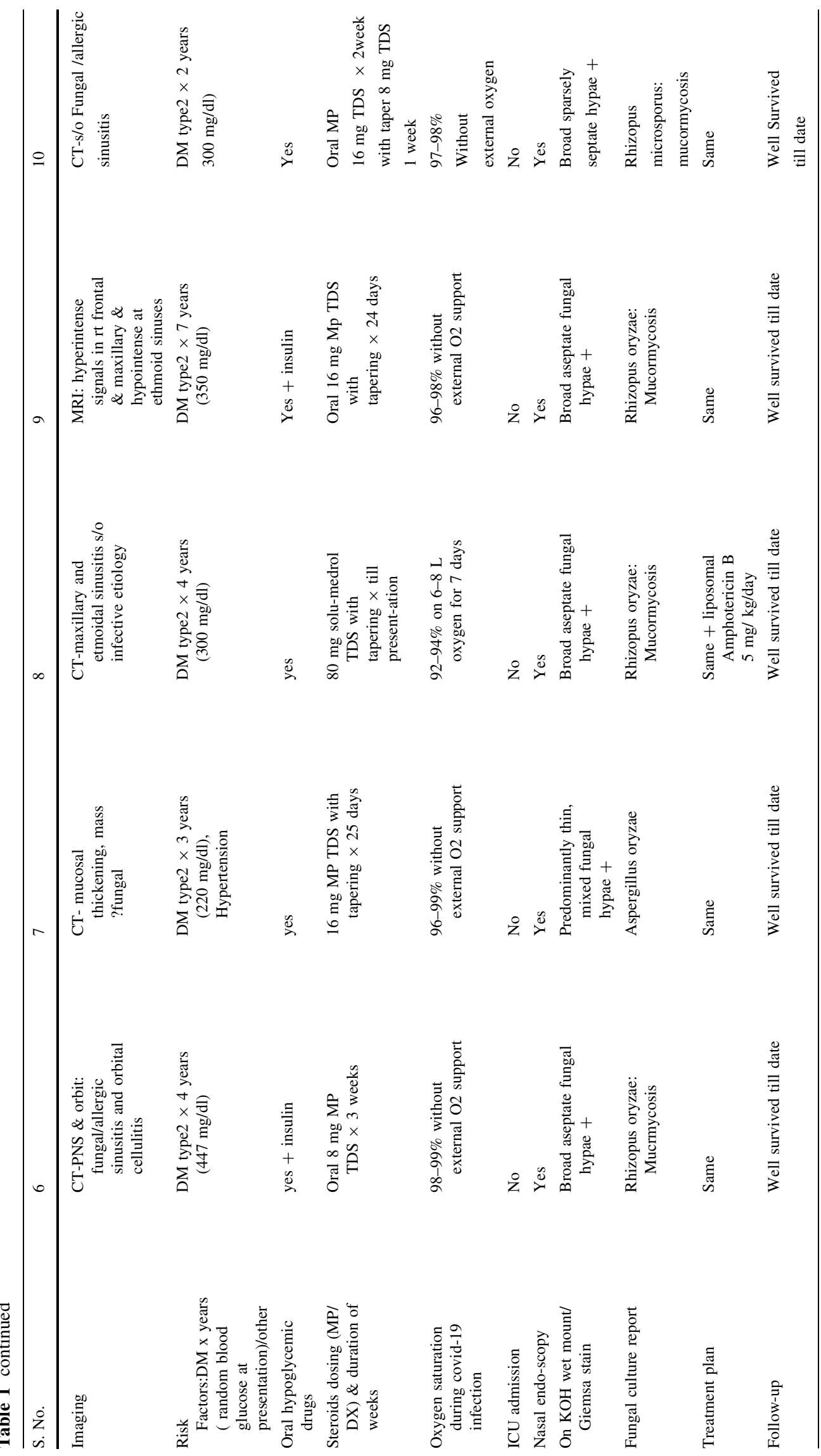




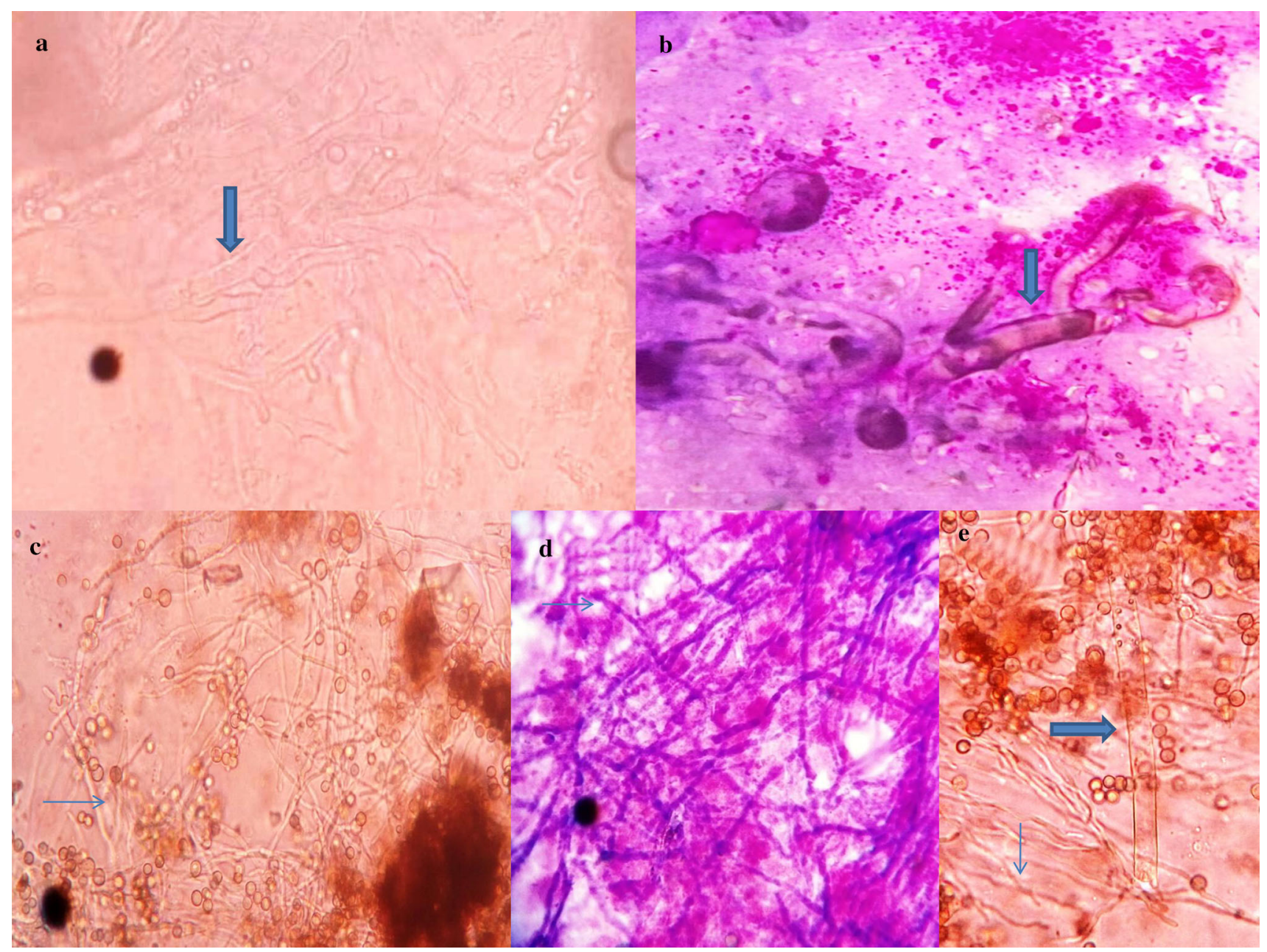

Fig. 3 a Smear showing broad, wide angled aseptate fungal hypae (thick arrow) possibly belonging to Mucormycosis (10\% KOH 40X). b Smear showing broad, wide angled aseptate fungal hypae (thick arrow) possibly belonging to Mucormycosis. Background shows inflammatory cells and necrosis (Giemsa 40X). c Smear showing thin, acute angled septate fungal hypae (thin arrow) possibly belonging to aspergillus $(10 \% \mathrm{KOH} 40 \mathrm{X})$. d Smear showing thin, acute angled

This may further suppressed their immunity causing opportunstic fungal infection. The prevailing guidelines in India recommends intravenous methyl prednisonone 0.5 $1 \mathrm{mg} / \mathrm{kg} /$ day or dexamethasone $0.1-0.2 \mathrm{mg} / \mathrm{kg} /$ day for moderate COVID-19 cases and $1-2 \mathrm{mg} / \mathrm{kg} / \mathrm{day}$ or $0.2-0.4 \mathrm{mg} / \mathrm{kg} / \mathrm{day}$ dexamethasone for severe cases for total of 5-10 days and doesn't recommend steroids for mild cases with $\mathrm{SpO} 2>94 \%$ at room temperature [16].

In the present article, the authors hypothesize that the trinity of COVID-19 infection, uncontrolled diabetic status and prolonged use of corticosteroids may act as cofactor for each-other causing vicious cycle for suppression of immunity allowing secondary fungal infection. In our article, the predominant symptoms were headache, nasal blockage, pain at local area, diminution of vision after few days of COVID-19 infection recovery. These features may septate fungal hypae (thin arrow) possibly belonging to aspergillus. Background contains few inflammatory cells (Giemsa 40X). e Smear showing predominant thin septate fungal hypae (thin arrow) with occasional sparsely broad (thick arrow) fungal hypae $(10 \% \mathrm{KOH}$ 40X)

be taken as initial warning symptoms in COVID-19 recovered patients for early suspicion of secondary fungal infection. Only 3 of 10 patients were given amphotericin B and 1of 10 patients was given posaconazole, So, we hypothesize that post-surgical antifungals are not necessarily required till the tissue infection is restricted below skull bone. All the patients survived till date without any further complication.

The simple routine $10 \% \mathrm{KOH}$ mount and Giemsa stain on crush biopsy smear may help for early diagnosis of fungal infection and to report the possible type of fungus for early management. Bala $\mathrm{K}$ et al showed in their study the utility of $\mathrm{KOH}$ wet mount for detection of mucormycosis [17]. The detection of fungal elements on Giemsa stain is rarely studied [18]. However, in cases where $\mathrm{KOH}$ mount may not help to visualize the type of fungal hypae 
clearly due to absence of colored staining, Giemsa stain may provide more light on type of fungal hypae to differentiate non-septate from septate hypae, to know mixed fungal and/or bacterial infection and to highlight the background.

\section{Conclusion}

Our study may be limited by short follow up duration but the authors primary aim was to report the characteristics of COVID- 19 recovered patients who presented with rhinoorbital mycoses. Patients should be made aware of strict glycemic control and limited use of corticosteroids. Patients should seek immediate medical attention if develops headache, pain, facial swelling, diminution of vision so that timely medical action may be taken for ensuring survival. Surgical debridement of nectrotic tissue is cornerstone of treatment which also provides sample for immediate fungal microscopy by $\mathrm{KOH}$ wet mount and Giemsa stain to provide quick possible diagnosis for timely management of the patients.

Funding There were no sources of funding (institutional, private and corporate financial support) for the work reported in this paper.

\section{Declaration}

Conflict of interest There was no conflict of interest either in designing and accomplishment of this study.

Consent for Publication The material has not been previously published or submitted elsewhere for publication.

Ethical Approval This manuscript has been read and approved by all the authors which represent our honest report of hard working.

Informed Consent Authors confirm that the statements of written informed consent from legally authorized representatives/parents/guardians are available.

\section{References}

1. Gorthi RS, Kamel G, Dhindsa S et al (2021) AACE Clinical Case Rep 7:6-92

2. Petrilli CM, Jones SA, Yang J, et al. 1966. Factors associated with hospital admission and critical illness among 5279 people with coronavirus disease 2019 in NewYork City: prospective cohort study. BMJ. 2020; 369(8249):m

3. Maini A, Tomar G, Khanna, et al . Sino-orbital mucormycosis in a COVID-19 patient: A case report. Int J Surg Case Rep 82 (2021) 105957
4. Dallalzadeh LO, Ozzello DJ, Liu CY, Kikkawa DO, Korn BS. 2021. Secondary infection with rhino-orbital cerebral mucormycosis associated with COVID-19. Orbit. Mar 23:1-4. https://doi.org/10.1080/01676830.2021.1903044. Epub ahead of print. PMID: 33752571

5. Sen M, Lahane S, Lahane TP et al (2021) Mucor in a viral land: a tale of two pathogens. Indian J Ophthalmol 69(2):244-252

6. These states have declared 'black fungus' a notifiable disease. Available from https://www.hindustantimes.com > india-news > these-st. Accessed on 9 june 2021

7. Paniker C, Editor. Ananthanarayan and Paniker's Textbook of microbiology. Orient Longman Publisher. 2006; p. 610-620

8. Over 28,200 'black fungus' cases recorded In India. Available from https://www.aa.com.tr > asia-pacific , over-28-200-blac. Accessed on 9 june 2021

9. Salehi M, Ahmadikia K, Badali H, Khodavaisy S. 2020. Opportunistic fungal infections in the epidemic area of COVID-19: A clinical and diagnostic perspective from Iran Mycopathologia; 185:607-11

10. Information about COVID-19 - WHO Official Information who.int

11. Chen G, Wu D, Guo W, Cao Y, Huang D, Wang H (2020) Clinical and immunologic features in severe and moderate Coronavirus Disease 2019. J Clin Invest 130:2620-2629

12. Moorthy A, Gaikwad R, Krishna S, Hegde R, Tripathi KK, Kale PG, Rao PS, Haldipur D, Bonanthaya K. 2021. SARS-CoV-2, Uncontrolled Diabetes and Corticosteroids-An Unholy Trinity in invasive fungal infections of the maxillofacial region? A retrospective, multi-centric analysis. J Maxillofac OralSurg. Mar 6:1-8. https://doi.org/10.1007/s12663-021-01532-1. Epub ahead of print. PMID: 33716414

13. Berbudi A, Rahmadika N, Tjahjadi AI, Ruslami R (2020) Type 2 diabetes and its impact on the immune system. Curr Diabetes Rev 16(5):442-449

14. Liu, Yan $\mathrm{MM}^{\mathrm{a}, \mathrm{b}}$; Yang, Yan $\mathrm{MM}^{\mathrm{a}, \mathrm{b}}$; Chen, Yalin $\mathrm{MM}^{\mathrm{a}, \mathrm{b}}$; Zhou, Linyue $\mathrm{MD}^{\mathrm{a}, \mathrm{b}}$; Xiong, Qian $\mathrm{MM}^{\mathrm{a}, \mathrm{b}}$; Xie, Chunguang $\mathrm{MD}^{\mathrm{a}}{ }_{*}$, 2020. The relationship between hyperglycemia and the infection of COVID-19 in diabetic patients, Medicine. 99(36) p e21806

15. Coutinho AE, Chapman KE. 2011. The anti-inflammatory and immunosuppressive effects of glucocorticoids, recent developments and mechanistic insights.Mol Cell Endocrinol. 335 (1):2-13

16. National clinical management protocol covid-19. Available from https://www.mohfw.gov.in > pdf > UpdatedDetail. Accessed on 12 june 2021

17. Bala K, Chander J, Handa U et al (2015) A prospective study of mucormycosis in north India: Experience from a tertiary care hospital. Medical Mycology 53(3):248-257

18. Zhang W, Yang H, Jiang L et al (1961) Techniques in the microscopic evaluation of corneal scrapings for diagnosis of fungal keratitis use of potassium hydroxide, giemsa and calcofluor white staining. J Int Med Res 2010(38):1961-1967

Publisher's Note Springer Nature remains neutral with regard to jurisdictional claims in published maps and institutional affiliations. 